\title{
Influence of Particle Shape on Microstructure of Granular Materials under Gravity
}

\author{
Beichuan Yan ${ }^{\mathrm{a}, *}$, Richard A. Regueiro ${ }^{\mathrm{a}}$ \\ ${ }^{a}$ Department of Civil, Environmental, and Architectural Engineering, University of \\ Colorado Boulder
}

\begin{abstract}
The paper presents a study of particle shape effect on microstructure within granular materials in gravitational environments using samples acquired from three-dimensional (3D) discrete element method (DEM) modeling and simulation $(\mathrm{M} \& \mathrm{~S})$ of gravitational pluviations. Three-axis ellipsoidal particles with various aspect ratios are examined for their spatial orientations, and the three-axis probability density functions (PDF) are analyzed, before and after pluviation. A complicated data structure, convex hull, is employed to construct tetrahedra that connect particle centroids, and investigate the internal topology and fabric of particle assemblies composed of various particle shapes. The Qhull-constructed connected tetrahedra within granular materials may serve as a useful tool to study the geometrical fabric (such as tetrahedron shape, solid angles, void ratio, etc), investigate particle collision requirements in sample preparation, describe strain/deformation/failure mechanisms, and explore stress distribution over granular materials.
\end{abstract}

Keywords: granular materials, particle shape and orientation, three-axis ellipsoid, discrete element method, constructed connected tetrahedron

\section{INTRODUCTION}

Particle shape plays an insurmountably-important role in determining micro-mechanical to macro-mechanical behavior of particle assemblies, and

\footnotetext{
*Corresponding author. Fax: +1-303-492-7317

Email addresses: beichuan.yan@colorado.edu (Beichuan Yan), richard.regueiro@colorado.edu (Richard A. Regueiro)
} 
their response to ambient conditions such as interaction with structures, gases and/or liquids. However, systematic studies of the particle shape effect on microstructure and macromechanical properties of granular materials using 3D DEM simulations are limited in the literature, due to various factors such as: (1) lack of realistic or generalized particle shape representations, analytically and numerically; (2) lack of computational capability, particularly an efficiently scalable parallel computing framework and algorithm; (3) lack of technical approaches to describe the microstructure of particle assemblies, for example, from fabric properties to average stress tensor over particle assemblies. Previous work is briefly summarized as below.

Nouguier-Lehon et al. (2003) performed two-dimensional discrete numerical simulations of biaxial tests on granular samples composed of different particle shapes (circular, isotropic polygonal and elongated polygonal particles), and found that particle shape highly influences the way anisotropy is generated during the deposition process under gravity. Ng (2004) used axi-symmetric ellipsoids in DEM simulations of triaxial tests, and found that mobilized friction angle decreases as the aspect ratio increases provided that void ratios of the two specimens are the same. The aspect ratio of an axisymmetric ellipsoidal particle was defined as the ratio of major axis length $a$ to minor axis length $b$, for which $a / b=1.2,1.5,1,7$ and 2 are used. Farhadi (2012) studied the rheological behavior of 2D shear-jammed granular systems by applying Couette shear in systems of bi-disperse disks and identical ellipses, and concluded that the slow relaxation towards an unjammed state in ellipses is associated with the possibility of small and slow changes in their orientations. Nguyen et al. (2015) adopted contact dynamics (CD) simulations to analyze the combined effects of polydispersity in particle size and shape for the quasistatic rheology of two-dimensional sheared granular media, where regular pentagons and irregular pentagons are used. The authors found that the shear strength is independent of the size span, but it unexpectedly declines with increasing shape polydispersity.

Yan et al. (2010) developed a contact resolution algorithm for three-axis ellipsoidal particles by constructing an extreme value problem of finding the deepest penetration of one particle into the other and solving it by an iterative eigenvalue method. The algorithm and its implementation have been shown to be robust such that it is applicable to not only regularly bulky ellipsoidal shapes but also high-aspect-ratio ellipsoidal particles such as disks and needles, as shown in Fig.1(a d). 
Peters et al. (2009) proposed a non-symmetric poly-ellipsoid shape which joins eight component ellipsoids in eight different octants respectively to produce continuous surface coordinates, normal directions and intersections. It is more computationally expensive than a symmetric ellipsoid, but it acts as a useful extension, as shown in Fig.1(e f). Other nonspherical particle representations include superellipsoids Wellmann et al. (2008); Delaney et al. (2010), superquadrics Williams and Pentland (1992), asymmetrical particles constructed by non-uniform rational basis splines (NURBS) Lim and Andrade (2014), arbitrary shapes using level set functions Kawamoto et al. (2016), etc.

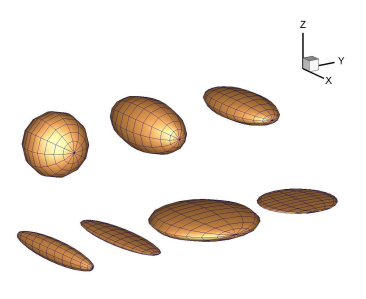

(a)

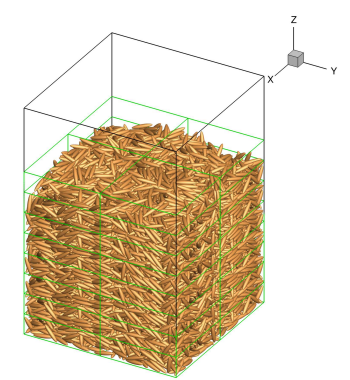

(d)

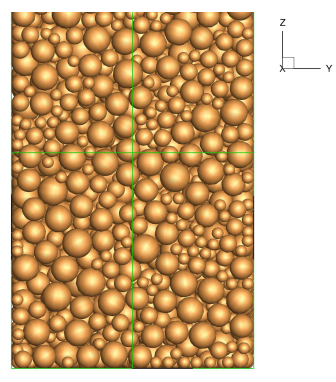

(b)

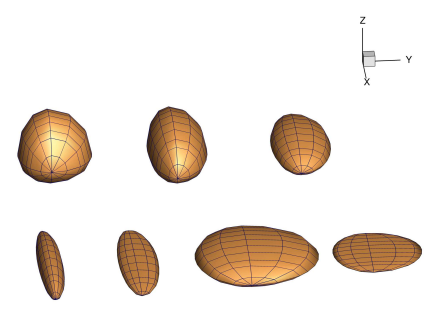

(e)

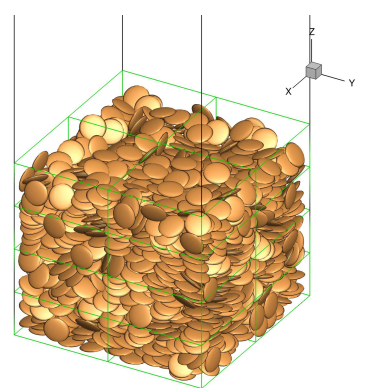

(c)

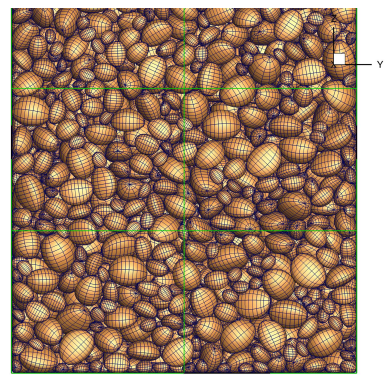

(f)

Figure 1: Ellipsoids and poly-ellipsoids represent a wide variety of shapes in DEM: (a) Ellipsoids with various aspect ratios; (b) Spherical particles; (c) Disk-like particles; (d) Needle-like particles; (e) Poly-ellipsoids with various aspect ratios; (f) Poly-ellipsoids.

Su et al. (2020) proposed a so-called "MAVL" number, $M=A / V \times L / 6$, to interpret 3D particle morphology $M$ as a function of the other geometry parameters, i.e., surface area $A$, volume $V$, and size $L$. The authors suggested the particle scale information can be reasonably related to the macroscopic mechanical property. It would be interesting to investigate such relationships 
using ellipsoidal and poly-ellipsoidal particles.

In this work, numerical results from the 3D DEM code ParaEllip3d Yan and Regueiro (2018a,b,c,d, 2019a,b,c) are presented to study the influence of particle shape on microstructure within granular materials in gravitational environments. Three-axis ellipsoidal particles with varying aspect ratios are adopted, which are not expected to mimic fully-realistic shapes, but instead, serve as a fundamental yet important abstraction of non-spherical particles. Section 1 has reviewed several particle shape representations in 3D DEM and the existence of particle shape effects; Section 2 clarifies several definitions in DEM simulations of gravitational pluviation; Section 3 provides visualization of ellipsoid three-axis orientations and analyzes probability density functions (PDF) before and after pluviation; Section 4 uses convex hull to construct tetrahedra that connect particle centroids to explore microstructure of particle assemblies; Section 5 presents void ratio of pluviated samples with different particle shapes; Section 6 illustrates stress distribution of pluviated samples with different particle shapes; Section 7 gives conclusion and outlook.

\section{DEM SIMULATIONS OF GRAVITATIONAL PLUVIATION}

Gravitational pluviation or raining of particles is simulated by 3D DEM to generate samples of packed particle assemblies: particles are initially "floated" in space without interaction and then gravitationally pluviated into a rigid container, as shown in Fig.2. At the end, all particles come to rest and are packed under gravity. A typical pluviation process is displayed at the following YouTube playlist Yan and Regueiro (2018b): https://www . youtube.com/playlist?list=PL0SpdOMtb6vU_7RwKbiBdpQGS7GGbBvUs.

\subsection{Pluviation initial velocity distribution}

In order to reduce computational cost of parallel computing, the "floated" particles are assumed to have a uniform and close spatial location distribution. However, the particles are assigned an initial velocity distribution to mimic the free fall velocity when they reach the "floated" state, i.e., each particle has an initial velocity that is reached by free fall from the top of the container to its current position. Four initial velocity distributions (IVD) are employed to evaluate the effect of interparticle collision intensity: $0 \mathrm{x}$ - the initial velocities are zero; $1 \mathrm{x}$ - normal free fall velocities; $2 \mathrm{x}$ - twice the $1 \mathrm{x}$ velocity distribution; and $3 \mathrm{x}$ - three times the $1 \mathrm{x}$ velocity distribution, shown 


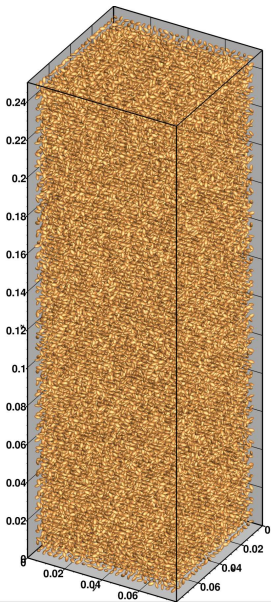

(a)

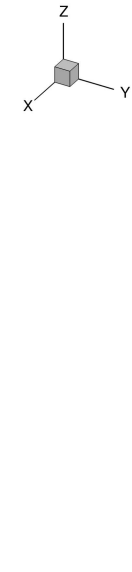

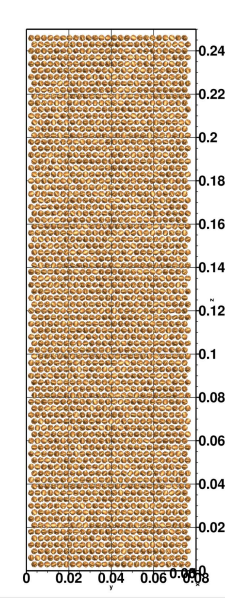

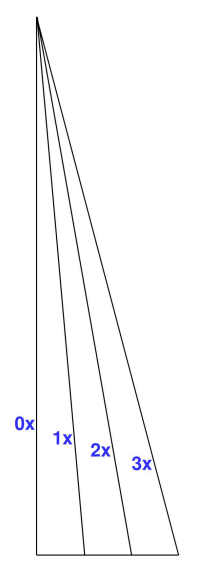

(b)

Figure 2: Gravitational pluviation and initial velocity distribution: (a) 3D view of initial particles; (b) Side view of initial particles and initial velocity distribution.

in Fig.2(b). These different IVD's serve two purposes: (1) approximate varying free fall heights and corresponding spatial velocity distributions to study the collision effect, but do not attempt to simulate the exact physical process of pouring particles into a container, which could vary by laboratory experimental approaches; (2) simplify the parallel computing process and maintain nearly the same minimal computational cost for varying collision intensities.

\subsection{Three-axis ellipsoid initialization}

A three-axis ellipsoid in 3D space is determined by the following parameters: position of the center $\left(x_{0}, y_{0}, z_{0}\right)$, lengths of three principal semiaxes $(a, b, c)$ and orientations of the three axes. The three-axis orientations are normally expressed as three unit vectors of direction cosine $(l 1, m 1, n 1)$, $(l 2, m 2, n 2),(l 3, m 3, n 3)$, respectively. Among the nine direction cosines only three of them are independent due to orthogonality condition between the three unit vectors. Euler angles $(\alpha, \beta, \gamma)$ are used to compute the nine direction cosines, as shown in Fig.3, where the axes of the global coordinate system (GCS) are denoted as x,y,z and the axes of local coordinate system (LCS) as X,Y,Z, and the line of nodes is defined as the intersection of the planes xy and XY (also written as $\boldsymbol{N}=\boldsymbol{z} \times \boldsymbol{Z}$ ), $\alpha \in[0,2 \pi]$ is the angle between the $\mathrm{x}$ axis and the $\mathrm{N}$ axis (so-called $\mathrm{x}$-convention), $\beta \in[0, \pi]$ is the angle between the $\mathrm{z}$ axis and the $\mathrm{Z}$ axis, and $\gamma \in[0,2 \pi]$ is the angle between 


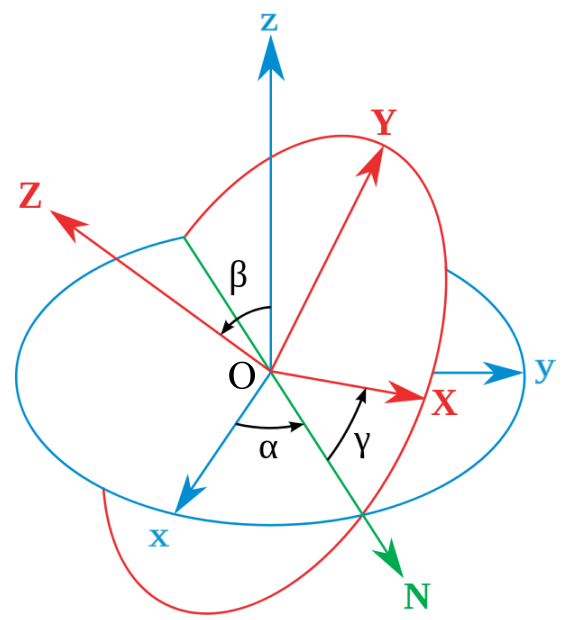

Figure 3: Definition of Euler angles.

the $\mathrm{N}$ axis and the $\mathrm{X}$ axis (so-called $\mathrm{x}$-convention).

The nine direction cosines are computed from the three Euler angles using the following equations:

$$
\begin{array}{r}
c_{1}=\cos \beta, c_{2}=\cos \alpha, c_{3}=\cos \gamma \\
s_{1}=\sin \beta, s_{2}=\sin \alpha, s_{3}=\sin \gamma \\
l_{1}=c_{2} c_{3}-c_{1} s_{2} s_{3}, m_{1}=s_{2} c_{3}+c_{1} c_{2} s_{3}, n_{1}=s_{1} s_{3} \\
l_{2}=-c_{2} s_{3}-c_{1} s_{2} c_{3}, m_{2}=-s_{2} s_{3}+c_{1} c_{2} c_{3}, n_{2}=s_{1} c_{3} \\
l_{3}=s_{1} s_{2}, m_{3}=-s_{1} c_{2}, n_{3}=c_{1}
\end{array}
$$

To generate a random orientation for a three-axis ellipsoid, three independent uniformly distributed random numbers are used to initialize the three Euler angles $\alpha, \beta$ and $\gamma$, then the nine direction cosines are computed by Eq. (1). It will be shown that only $n_{3}$ of the nine direction cosines holds uniform distribution while others do not.

\subsection{Three-axis ellipsoid shapes}

A three-axis ellipsoidal particle contains three independent semi-axis lengths (major semi-axis $a$, middle semi-axis $b$, and minor semi-axis $c$, assuming $a \geq b \geq c$ ), or two independent aspect ratios: 1st aspect ratio $(A R 1=b / a)$, and 2 nd aspect ratio $(A R 2=c / a)$. Eight samples of various combinations of $A R 1$ and $A R 2$ are used to represent ellipsoidal shapes of 
interest, as shown in Table 1. In pluviation simulations, the only difference between the 8 samples lies in the particle shapes, while other factors such as initial spatial distribution, initial three-axis orientations, initial velocity distribution, etc, are exactly the same for purpose of comparison.

Each sample consists of 51,250 uniformly-sized particles. The major semiaxis length of each particle is $1.5 \mathrm{~mm}$, and the middle and minor semi-axis lengths vary according to Table 1 . The rigid container that holds particles during pluviation is $8 \mathrm{~cm} \mathrm{x} 8 \mathrm{~cm} \times 24 \mathrm{~cm}$ in $\mathrm{x}, \mathrm{y}$, z directions, respectively. The DEM pluviation simulation parameters are listed in Table 2.

Table 1: Eight ellipsoidal particle shapes (the perspective angle is defined in Section 4).

\begin{tabular}{ccccc}
\hline sample & $a(\mathrm{~mm})$ & $b / a$ & $c / a$ & persp. angle $(\mathrm{deg})$ \\
\hline 1 & 1.5 & 1.0 & 1.0 & 35 \\
2 & 1.5 & 0.9 & 0.7 & 47 \\
3 & 1.5 & 0.9 & 0.6 & 52 \\
4 & 1.5 & 0.8 & 0.6 & 57 \\
5 & 1.5 & 0.8 & 0.5 & 59 \\
6 & 1.5 & 0.7 & 0.5 & 64 \\
7 & 1.5 & 0.7 & 0.4 & 67 \\
8 & 1.5 & 0.6 & 0.4 & 68 \\
\hline
\end{tabular}

Table 2: Numerical parameters used in pluviation simulation.

\begin{tabular}{|l|c|}
\hline Young's modulus $E(\mathrm{~Pa})$ & $4.5 \times 10^{10}$ \\
\hline Poisson's ratio $\nu$ & 0.25 \\
\hline specific gravity $G_{s}$ & 2.65 \\
\hline coef. of friction $\mu$ & 0.5 \\
\hline contact damping ratio $\xi$ & 0.85 \\
\hline particle max. radii $(\mathrm{mm})$ & 1.5 \\
\hline particle shape $($ aspect ratio) & $1: 1: 1$ to $1: 0.6: 0.4$ \\
\hline time step $\triangle t(\mathrm{sec})$ & $2.0 \times 10^{-7}$ \\
\hline
\end{tabular}

\section{PARTICLE THREE-AXIS ORIENTATIONS}

A side view of the mid-plane slice of the 8 samples acquired from pluviation is illustrated in Fig.4. Firstly, it is seen that the samples possess 
different volumes in their packed state: the lower the aspect ratios (i.e., the flatter the ellipsoids), the smaller the packed volume; secondly, the particle assembly fabric can be observed from the slice view: the lower the aspect ratios, the more horizontally inclined the particles. It should be noted that the fabric distribution trend can only be observed from slice views across the particle assembly bodies, whereas it is not seen from side view of the whole particle assembly, due to mechanical interaction between container side walls and nearby particles.

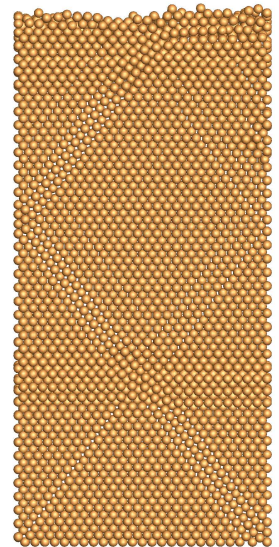

(1)

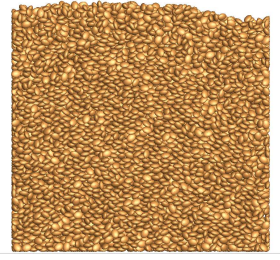

(5)

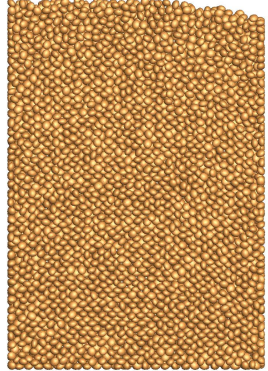

$(2)$

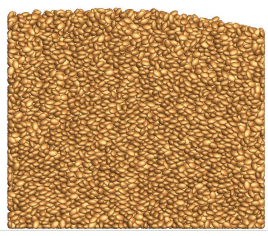

(6)

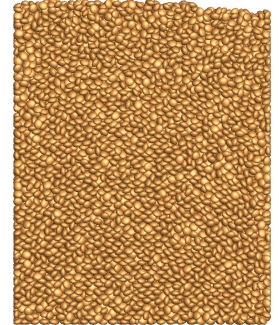

$(3)$

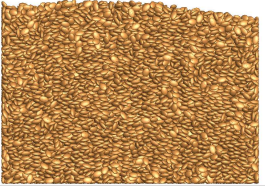

(7)

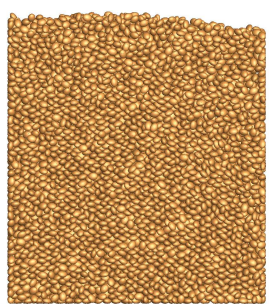

$(4)$

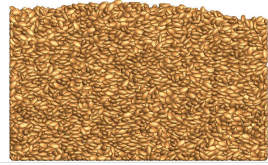

(8)

Figure 4: Slice view of the 8 samples obtained from pluviation simulations.

Figure 5 plots the major axis orientations using a side view of the 8 samples, which confirms the visual impression of fabric distribution trend. Side view of the minor axis orientations is illustrated in Fig.6, from which it is seen that more minor axes tend to be vertically aligned for lower aspect ratios.

Figure 7 plots the initial probability density function (PDF) of angles between the three ellipsoid axes and three planes (yz plane, zx plane, xy 


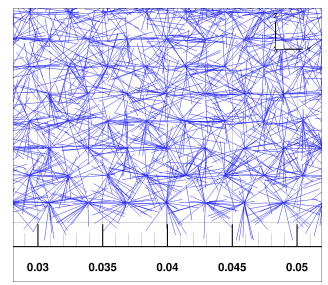

(1)

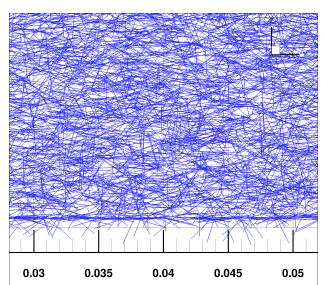

(5)

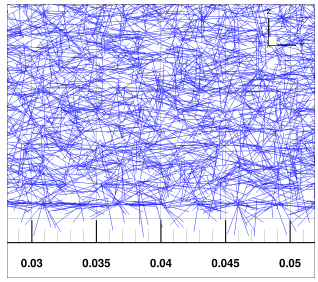

(2)

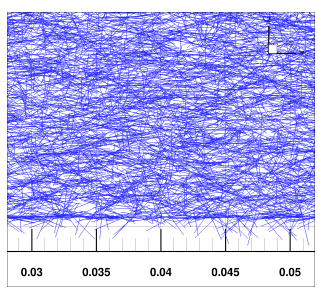

(6)

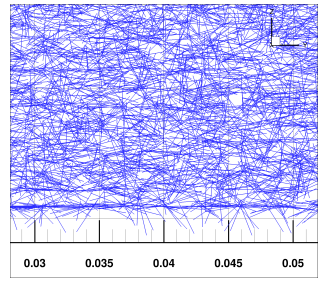

(3)

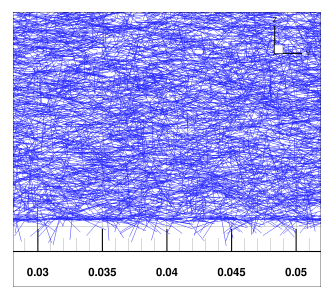

(7)

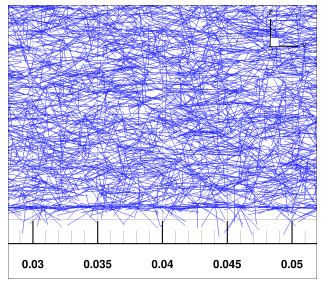

(4)

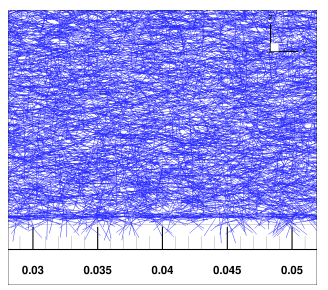

(8)

Figure 5: Side view of major axis orientations of the 8 samples.

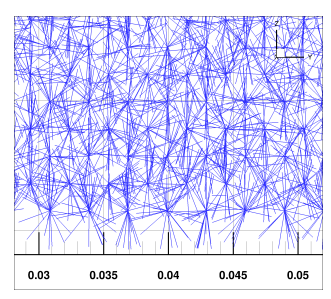

(1)

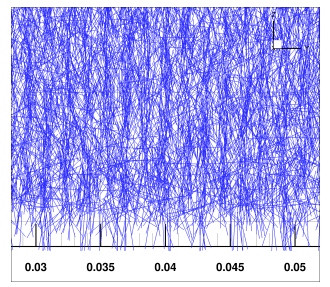

(5)

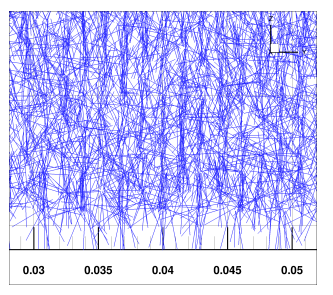

(2)

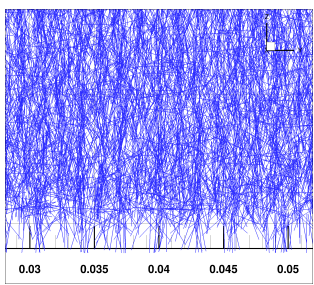

(6)

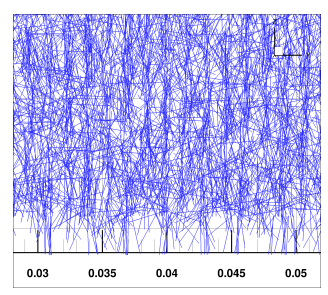

(3)

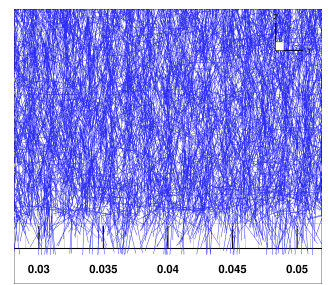

(7)

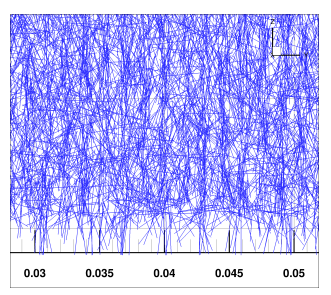

(4)

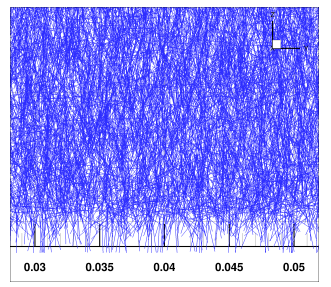

(8)

Figure 6: Side view of minor axis orientations of the 8 samples. 
plane), respectively. In terms of Eq. (1), only $n_{3}$ of the nine direction cosines holds uniform distribution, which is verified by the lower-right graph in Fig.7. For comparison, the PDFs of gravitationally-pluviated samples \#1 and \#2 are plotted in Figures 8 and 9, respectively. Three evident changes are noticed in the process of pluviation:

- the PDF spikes associated with very small angles between minor axis and vertical yz and zx planes disappear, i.e., minor axes lose their initial vertical alignment during pluviation;

- the PDF spikes associated with very small angles between major/middle axes and horizontal xy plane disappear, i.e., major/middle axes lose their initial horizontal alignment during pluviation;

- the initial uniform distribution of $n_{3}$, which equals the cosine of the complementary angle of minor axis w.r.t horizontal plane, has changed to a plateau in sample $\# 1$ (spheres), i.e., the $-90 \sim-70$ and $70 \sim 90$ degree portions are substantially reduced. Whereas the $-70 \sim+70$ degree portions are also moderately reduced for ellipsoids.

- the PDFs are obviously affected by particle aspect ratios if comparing the angles between minor axis and xy plane.

Figure 10 plots the probability density function (PDF) of angles between ellipsoid minor axes and horizontal xy planes for the 8 gravitationallypluviated samples. It is noticed that:

- except for the spherical particle assembly exhibiting a plateau shape, all of the ellipsoidal particle assemblies produce a concave-plateau PDF;

- the $-90 \sim-70$ and $70 \sim 90$ degree portions are substantially reduced for both spheres and ellipsoids in comparison to the initially uniform distribution, which reveals again that ellipsoid minor axes cannot sustain initial vertical alignment during pluviation;

- for ellipsoidal particles, the minor axes lose their horizontal 0 degree portion while gaining $50 \sim 80$ degree portion, namely, the standing-onside portion decreases and horizontally-inclined portion increases for minor axes;

- the flatter the ellipsoids (i.e., with smaller $A R 1$ and $A R 2$ ), the more horizontally-inclined, which is seen from PDFs of the 8 samples. 

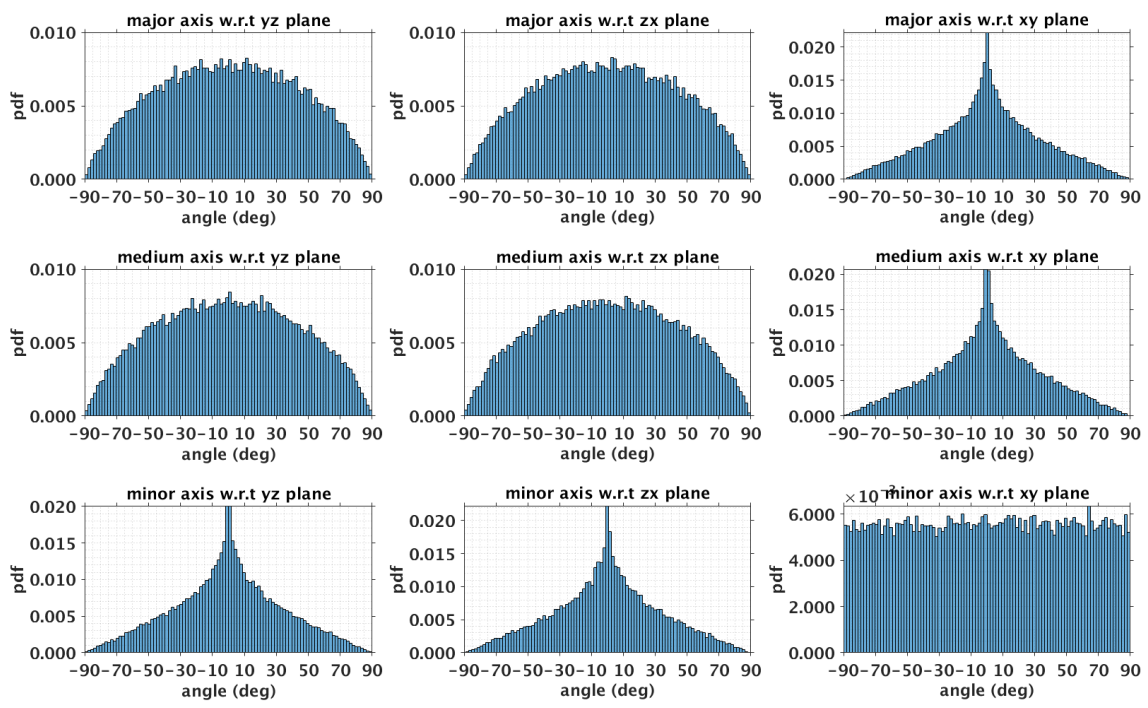

Figure 7: Initial three-axis PDF of all samples.
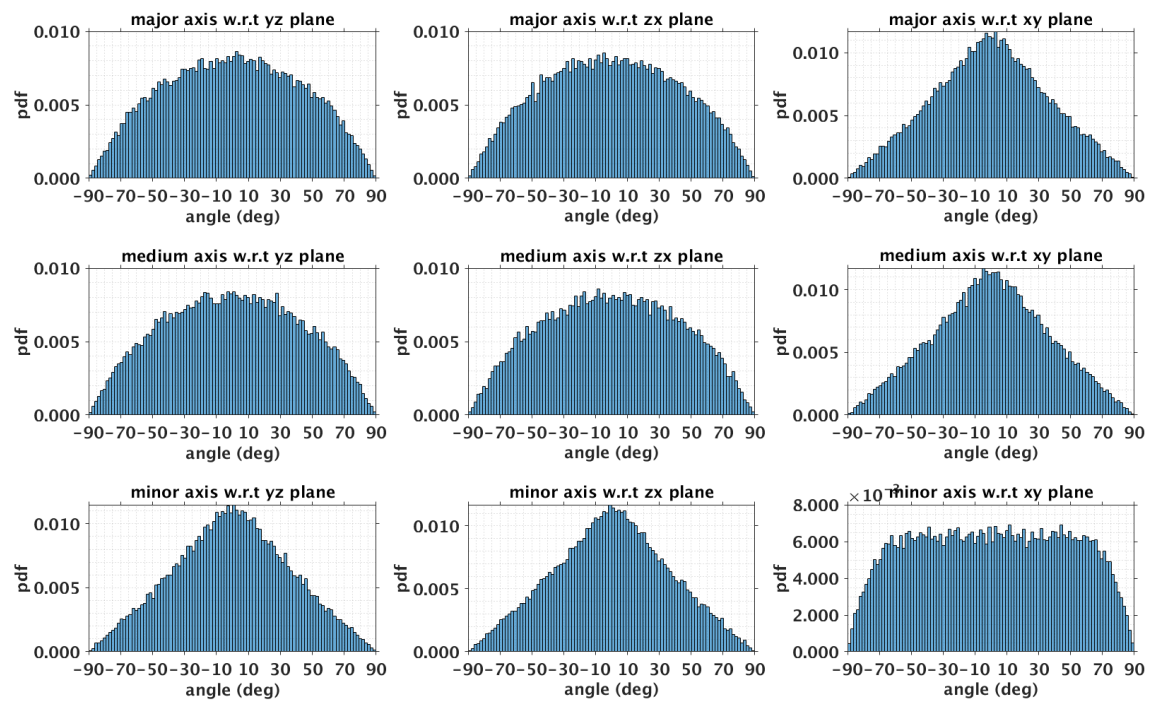

Figure 8: Three-axis PDF of pluviated sample \#1. 

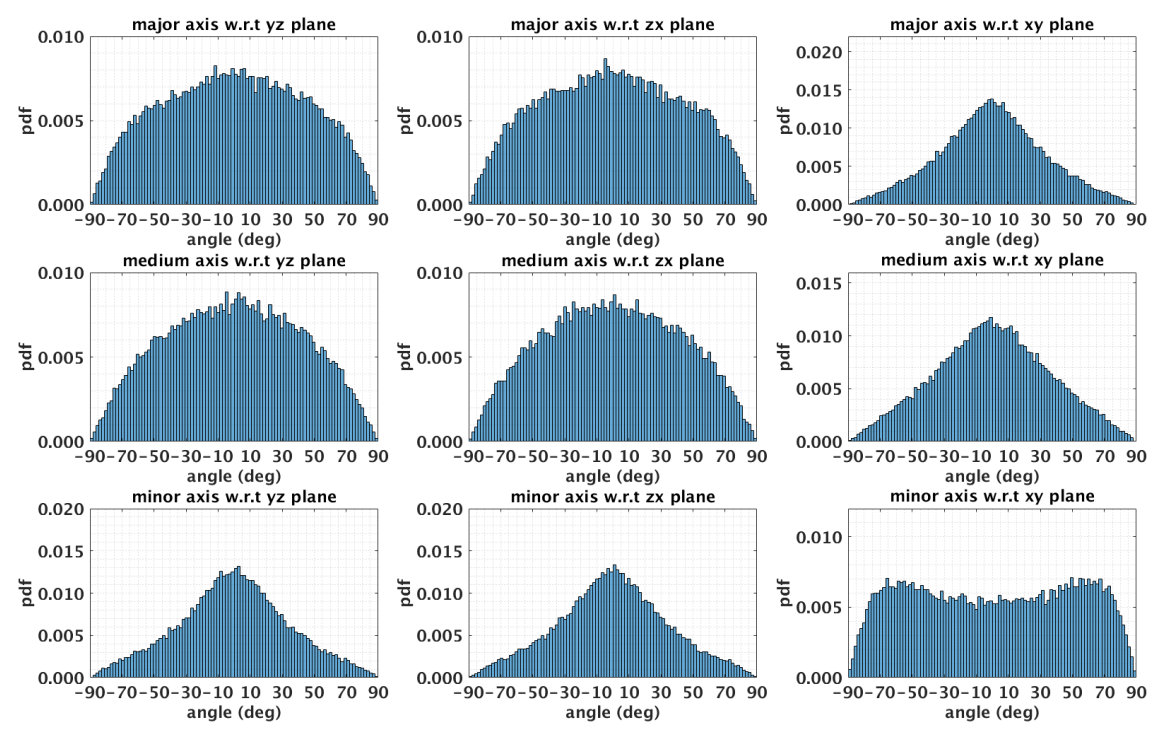

Figure 9: Three-axis PDF of pluviated sample \#2.

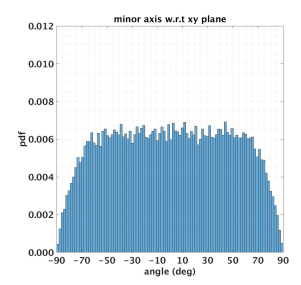

(1)

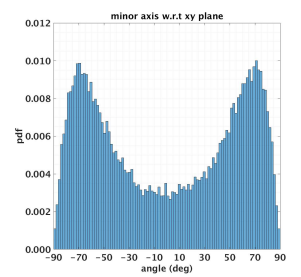

(5)

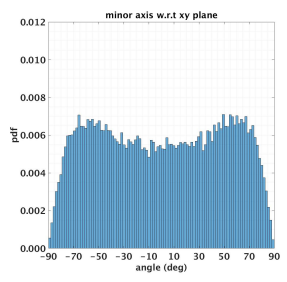

(2)

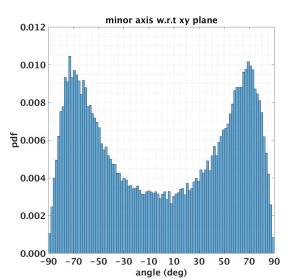

(6)

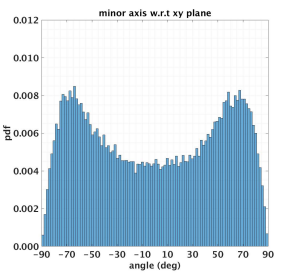

(3)

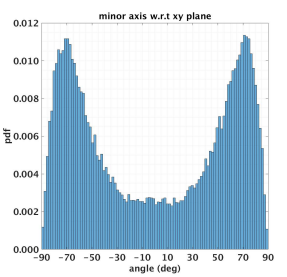

(7)

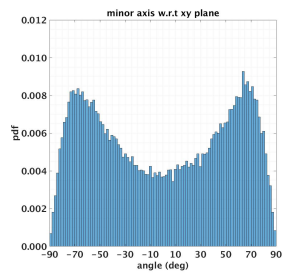

(4)

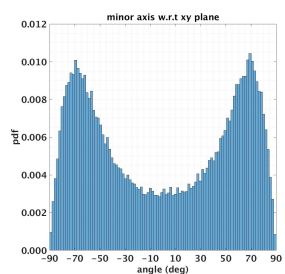

(8)

Figure 10: Minor axis PDF of the 8 samples. 


\section{TETRAHEDRA CONNECTING PARTICLE CENTROIDS}

A complicated data structure, convex hull, is employed within ParaEllip3d to explore the internal topology and fabric of particle assemblies composed of various particle shapes. The open source code, Qhull (www.qhull. org), is utilized for this purpose: Qhull implements the Quickhull algorithm and computes the convex hull, Delaunay triangulation, Voronoi diagram, etc.

Qhull computes the Delaunay triangulation by computing a convex hull, which enables construction of tetrahedra in 3D space that connect the centroids of every four neighboring particles, or the contact points of every four neighboring interparticle contact pairs, and examines important geometric quantities such as tetrahedron shape, solid angles, dihedral angles, etc; especially, Qhull makes it possible to track geometrical deformation within dislocation bands in pluviation simulations or shear bands in triaxial test simulations using constructed tetrahedra; furthermore, Qhull provides the possibility to compute finite strain of a particle assembly by tracking motion of representative individual particles.

Figure 11(a) shows the side view of 7,000 gravitationally-pluviated spherical particles, and Fig.11(b) illustrates the side view of tetrahedra constructed by connecting particle centroids. It should be noted that Qhull may generate a certain degree of noise, i.e., connecting particles that are not close to each other. We have developed two types of convex hull filters: (i) contact number filter, which screens out tetrahedra that have less than a certain number of interparticle contact pairs. This type of filter requires DEM interparticle contact information; (ii) distance filter, which screens out tetrahedra that have more than a certain relative gap ratio between any two particles located at tetrahedron vertices.

A constructed tetrahedron has a maximum of six interparticle contact pairs from the four particles at its vertices. Figure 11(c) plots the tetrahedra that have at least 4 contact pairs, and Fig.11(d) plots the tetrahedra that have at least 5 contact pairs, in which the number of such tetrahedra is merely around 20. It is surprising to find that no tetrahedron possesses 6 interparticle contacts for the 7,000 spherical particles, which indicates that the dynamic pluviation process generates adequate collision between particles and does not produce highly dense microstructures.

Figure 12 presents a small patch side view of all tetrahedra constructed for each of the 8 samples. It is discovered that the tetrahedron edges concentrate to a certain extent and form a perspective pattern for each sample. Obviously 


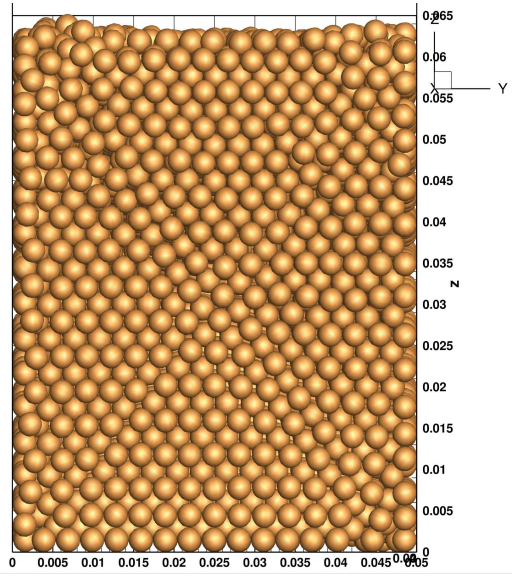

(a)

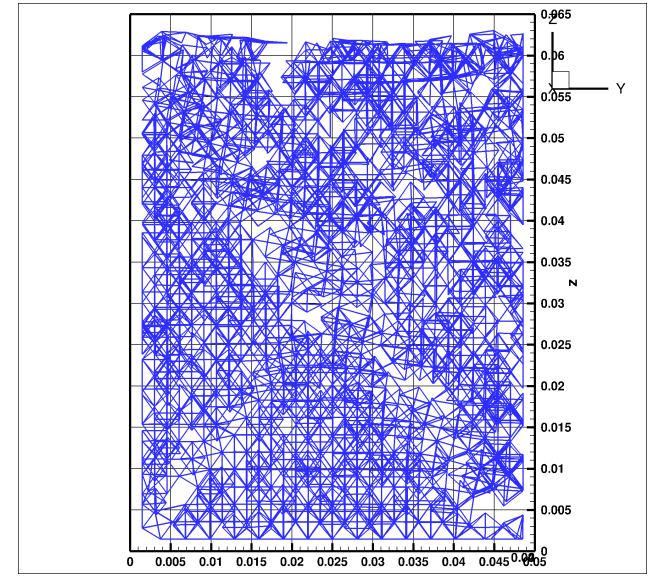

(c)

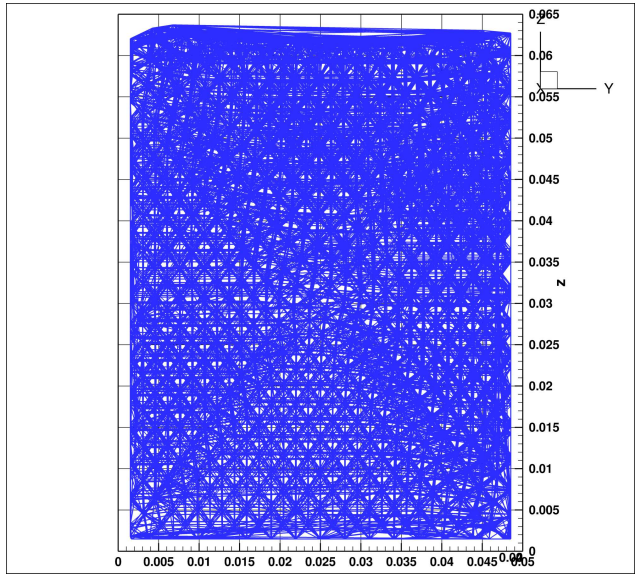

(b)

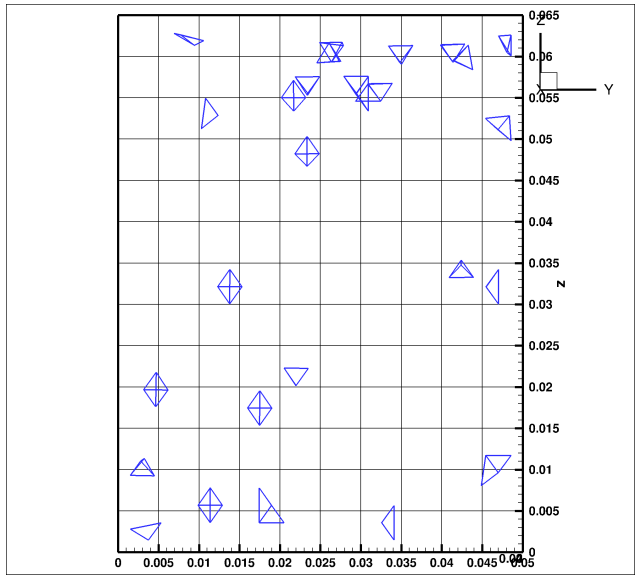

(d)

Figure 11: Side view of tetrahedra constructed from 7k spherical particles: (a) 7k particles; (b) tetrahedra; (c) tetrahedron filter by contact number 4; (d) tetrahedron filter by contact number 5 . 
the perspective patterns, particularly the angles between the "concentrated" lines, are related to particle shapes. We define half of the top angle between crossing concentrated lines as "perspective angles", as shown by the red lines in Fig.12. The measurements of perspective angles are listed in Table 1 in Section 2. It is evident that the lower the aspect ratios (i.e., the flatter the ellipsoids), the larger the angles. The relationship between perspective angles and particle shapes is worthy of further investigation.

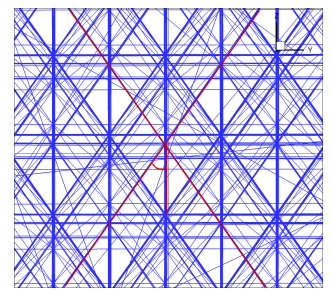

(1)

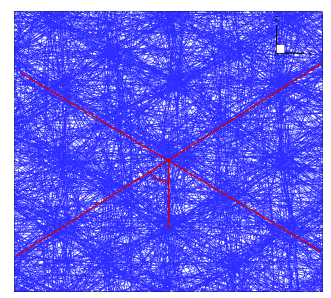

(5)

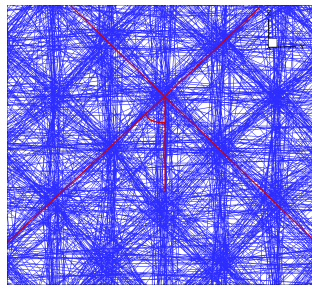

$(2)$

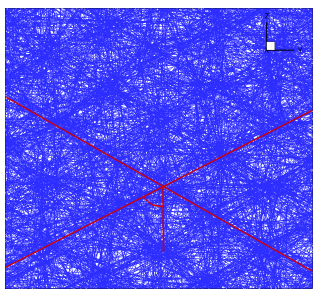

(6)

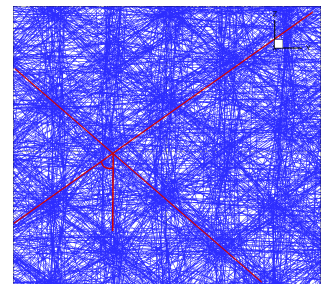

(3)

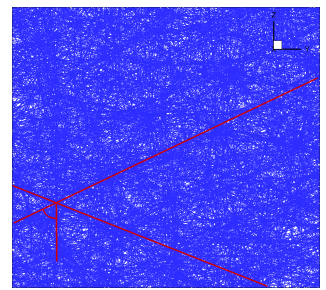

(7)

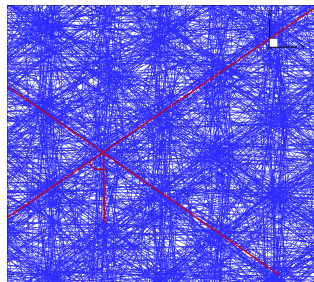

$(4)$

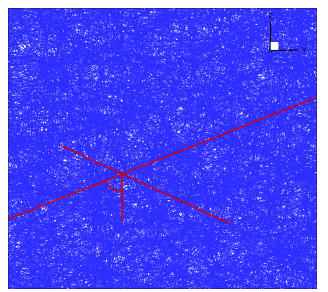

(8)

Figure 12: Side view of tetrahedra constructed from 51,250 particles of the 8 samples.

The formation of "concentrated" lines is interpreted by Fig.13, where (a) is an extracted column of the particles and (b) is the side view of the tetrahedra. Figure 13(c) shows a 3D view of the tetrahedra, where the tetrahedron structure is observed clearly. The "concentrated" lines are actually tetrahedron edges that are "overlapped" from a perspective view.

Figure 14(a) plots average values of tetrahedron top solid angles (in steradians) of the 8 samples for 4 different initial velocity distributions (IVD), and Fig.14(b) plots the equivalent cone angles (in degrees). A solid angle is a 3D measure of the amount of the field of view from a particular point that a given object covers, referring to https://en.wikipedia.org/wiki/Solid_ angle. Hereby the tetrahedron top solid angle is the field of view from the top vertex that the other three vertices cover; and the equivalent cone angle can be regarded as the angle in $2 \mathrm{D}$ slice view of the $3 \mathrm{D}$ solid angle. The solid angle (or cone angle) decreases and converges for each sample as the 


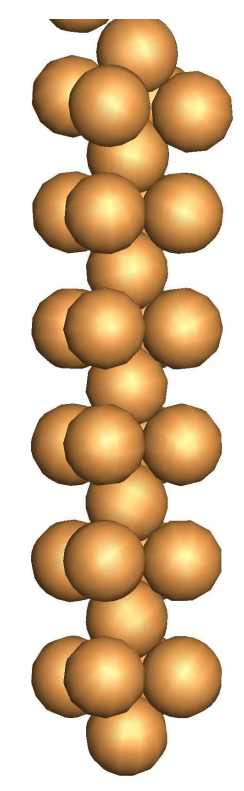

(a)

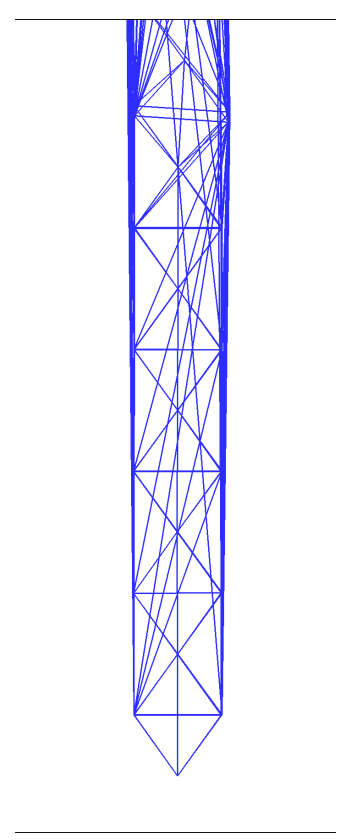

(b)

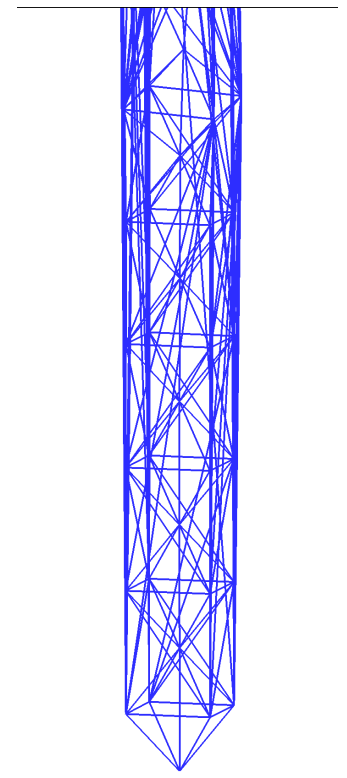

(c)

Figure 13: Illustration of the "concentrated" lines: (a) Extracted column of particles; (b) Side view of the tetrahedra; (c) 3D view of the tetrahedra. 
IVD increases: a clear gap exists between IVD $0 \mathrm{x}$ and IVD $1 \mathrm{x}$ to $3 \mathrm{x}$, and IVD $1 \mathrm{x}$ to $3 \mathrm{x}$ produce very close results. Hereby a conclusion can be drawn: the microstructure or fabric of particle assemblies can be regarded identical when IVD is high enough to produce adequate collisions between particles in pluviation. The conclusion will be tested against macroscopic variables such as void ratio later. Regarding the trend of the angles, it is seen that the aspect ratio 10-8-6 achieves the highest solid angle and cone angle. The interpretation of this phenomenon needs further work, for example, by applying convex hull filters to eliminate tetrahedra that connect not-near-enough particles through Qhull algorithm.

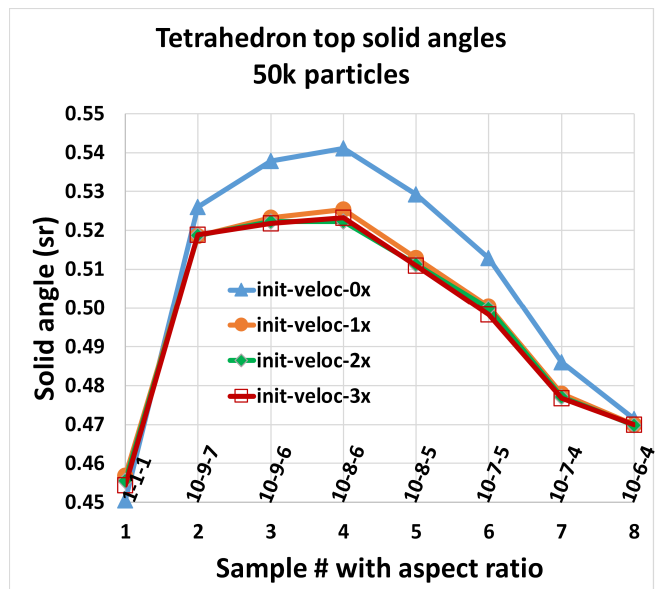

(a)

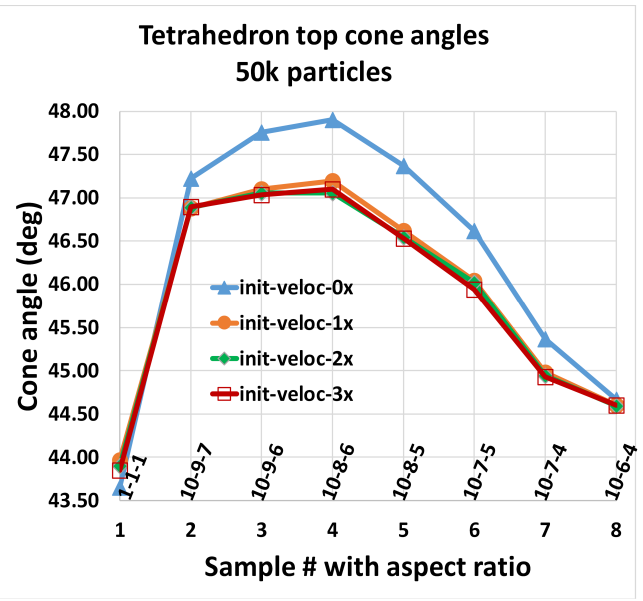

(b)

Figure 14: Average solid angles and cone angles of the 8 samples: (a) Average solid angles; (b) Average cone angles.

The average tetrahedron volumes are plotted for each sample in Fig.15(a), whereby the volume decreases with lower aspect ratios. The relative volume, namely, ratios of tetrahedron volume to ellipsoidal particle volume are presented in Fig.15(b), from which the IVD effect is observed again. Combining Fig.14 and Fig.15, it is observed that spheres differ significantly from the family of 8 ellipsoids in terms of the microstructural quantities.

Figure 16 plots average tetrahedron volume with respect to individual particle volume, or product of aspect ratios $A R 1$ and $A R 2$, since the maximum semi-axis length is constant for a sample. They exhibit a nearly linear relationship, indicating the tetrahedron volume and particle shapes are closely associated. 


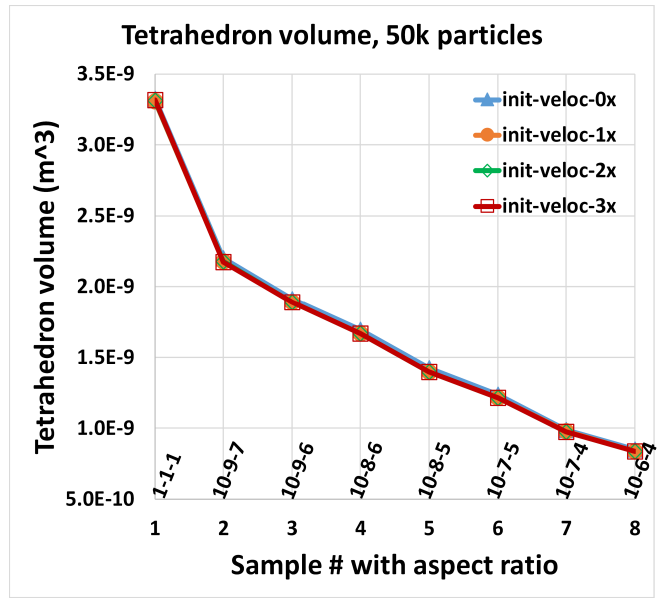

(a)

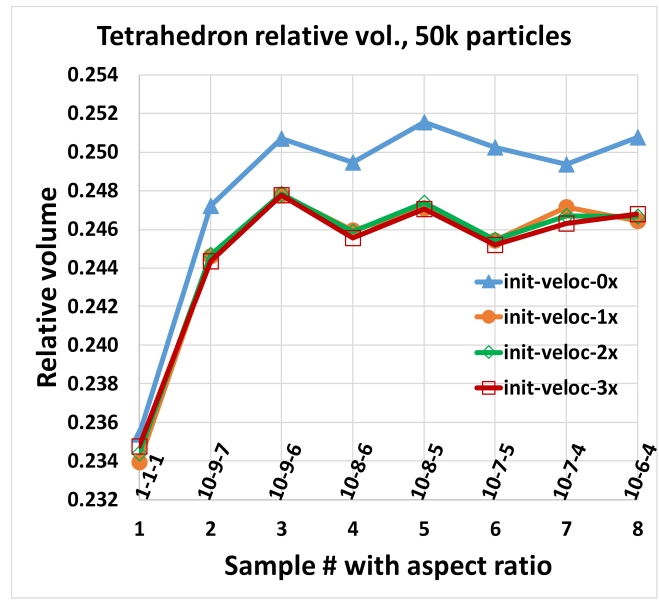

(b)

Figure 15: Tetrahedron volume of the 8 samples: (a) Average tetrahedron volumes; (b) Relative tetrahedron volumes.

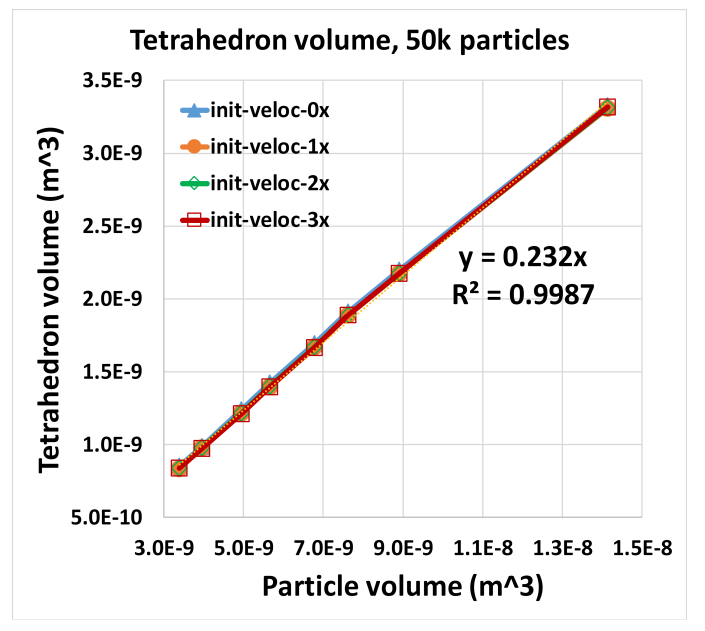

Figure 16: Tetrahedron volume vs particle volume.

\section{VOID RATIO}

The packed state of different particle shapes shown in Fig.4(a) may lead to an impression that particles with lower aspect ratios possess a lower void ratio. In this section, void ratio is measured by trimming away top particles with a horizontal flat surface. Elevation of the trimming surface may be shallow or deep with respect to the top of the samples, which is shown to 
have negligible effect in measuring void ratio. Figure 17 plots void ratio of the 8 samples at different IVD conditions. It is seen that:

Figure 17(b) plots the void ratio with regard to the product of aspect ratios $A R 1$ and $A R 2$, which exhibits a nonlinear relationship. A further study of the relations between void ratio and $A R 1$ and $A R 2$, namely, finding a multivariable function, will be carried out in the future.

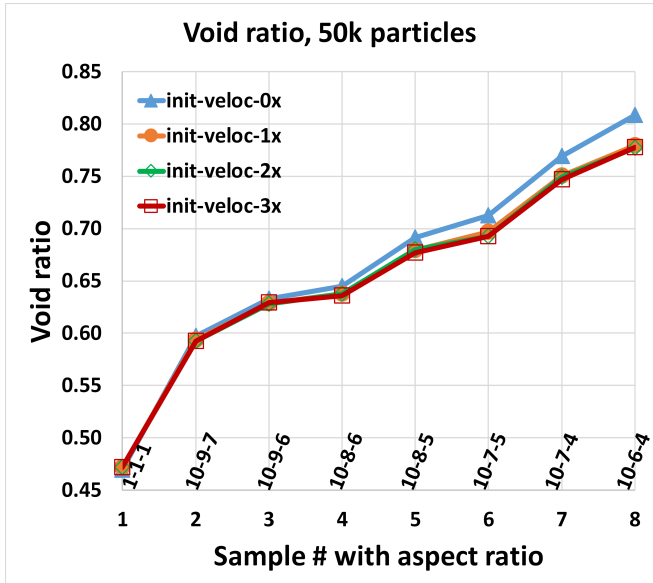

(a)

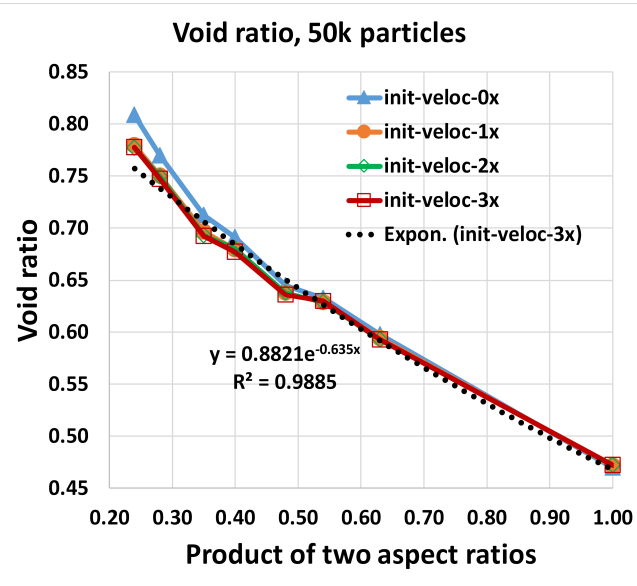

(b)

Figure 17: Void ratio of the 8 samples: (a) sample number with aspect ratio; (b) product of two aspect ratios.

1. the void ratio ranges from 0.46 to 0.81 . Our particle sizes fall within the range of coarse sand according to Craig's Soil Mechanics, 8th edition Knappett and Craig (2012); O'Sullivan (2013). Usually, low density sand typically has a void ratio of about 0.8 , while high density sand with angular particles typically has a void ratio about 0.4. As a comparison, FCC crystal structures have void ratio of 0.26 in unit cell, and BCC crystal structures have a value of 0.32 .

2. the lower the aspect ratios (i.e., the flatter the ellipsoids), the higher the void ratio. This is a fundamental yet important conclusion that associates void ratio with particle shape. It can be interpreted as follows: flatter particles usually produce higher rolling resistance between them in the process of gravitational pluviation, and cannot achieve as dense interparticle contacts as spheres. 
3. the IVD effect is observed again, i.e., strong enough collisions between particles during pluviation result in mechanically equivalent samples.

\section{STRESS}

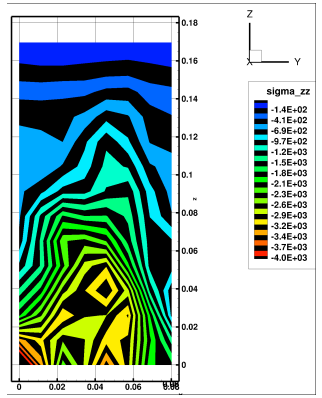

(1)

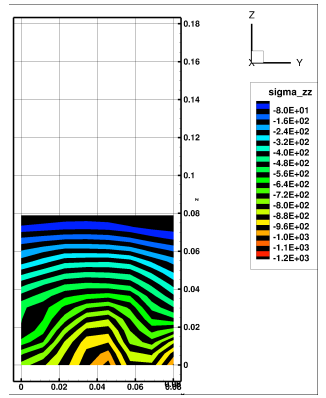

(5)

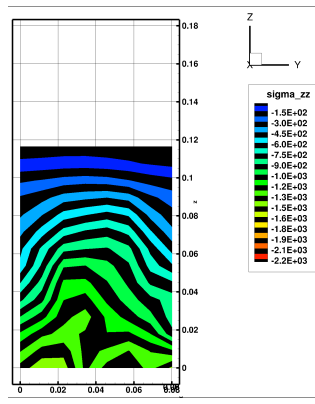

(2)

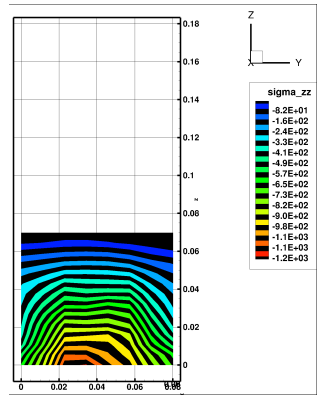

(6)

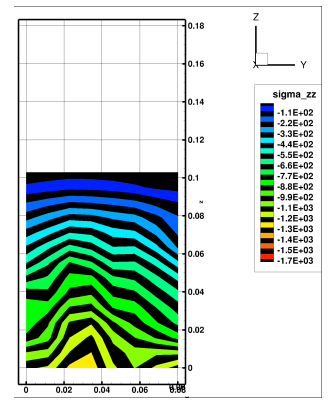

(3)

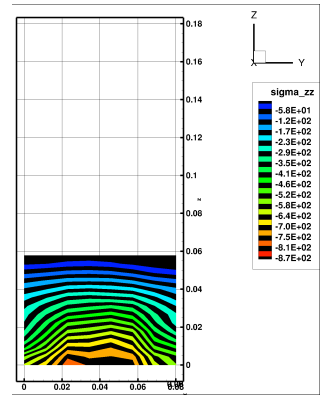

(7)

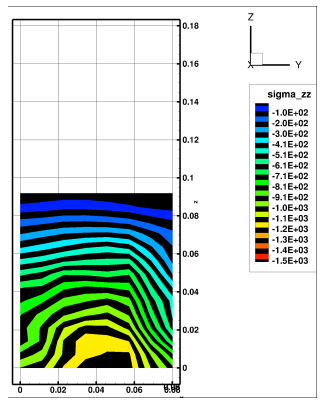

(4)

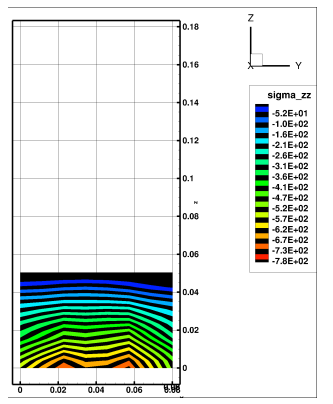

(8)

Figure 18: Slice view of $\sigma_{z z}$ distribution of 8 samples.

Figure 18 plots stress $\sigma_{z z}$ distribution of the 8 samples at rested state acquired from pluviation, in terms of Bagi's formula for stress tensor for granular materials Yan and Regueiro (2019b,c). The stress exhibits non-uniform spatial distributions under static equilibrium, mostly a dome shape in 3D space, which is related to particles shapes of the sample and boundary effect of the container. This phenomenon reveals a microstructural effect on stress distribution within granular materials that is attributed to their discrete particulate nature, namely, particle size, shape and boundary conditions. The dome-shaped spatial stress distribution is associated with conducting pluviation in a relatively narrow container, which prevents particles from moving laterally, locking up different microstructures along horizontal levels. A wider 
container allows more lateral motion of the particles and alleviates the lockup effect along its side walls, resulting in a more evenly distributed stress contour Yan and Regueiro (2019c).

\section{CONCLUSION AND OUTLOOK}

Based on the numerical simulations in the paper, the following conclusions can be made:

- All of the ellipsoidal particle assemblies produce a concave-plateau shape of PDF for angle between ellipsoid minor axes and horizontal planes during gravitational pluviation, even though the angle is initially set a uniform distribution using random number generator.

- Regarding the axis orientations, the flatter the ellipsoidal particles, the more horizontally-inclined.

- With convex hull algorithm, it is possible to construct connected tetrahedra that link particle centroids within a particle assembly.

- The connected tetrahedra in 3D space provide useful information on microstructure or fabric of a particle assembly. The flatter the particles, the large the "perspective" angle.

- Strong enough collisions between particles during pluviation result in mechanically equivalent samples, i.e., the particles need to own a minimal initial velocity computed by free fall from the top of the container, numerically.

The following extensions are worthy of further study: (1) define shape tensors that describe three-axis orientations and apply them for the study of anisotropy of granular materials in gravitational environments; (2) compute fabric tensor and compare it with shape tensors, to see which better describe the fabric of granular materials, or how they may work together for that purpose; (3) study the relations between void ratio of a particle assembly and the aspect ratios of ellipsoidal particles using a complete dot matrix of $A R 1$ and $A R 2$, namely, find void ratio distribution as a multivariable function of $A R 1$ and $A R 2$; (4) develop algorithms to compute finite strain of a particle assembly by tracking motion of connected tetrahedra constructed by convex hull. 


\section{Data Availability Statement}

Some data, models, or codes that support the findings of this work are available from the corresponding author upon reasonable request. The codes are available from this website: https://gitlab.com/micromorph/ paraellip3d-cfd

\section{Acknowledgments}

Funding for this work was provided by ONR grant N00014-17-1-2704, which is gratefully acknowledged. Computational resources were provided by the DoD High Performance Computing Modernization Program (HPCMP). We declare that there is no conflict of interest.

Gary W Delaney, Paul W Cleary, Matt D Sinnott, and Rob D Morrison. Novel application of dem to modelling comminution processes. In IOP Conference Series: Materials Science and Engineering, volume 10, page 012099. IOP Publishing, 2010.

Somayeh Farhadi. Shape Effects on Jamming of Granular Materials. PhD thesis, Duke University, 2012.

Reid Kawamoto, Edward Andò, Gioacchino Viggiani, and José E Andrade. Level set discrete element method for three-dimensional computations with triaxial case study. Journal of the Mechanics and Physics of Solids, 91: $1-13,2016$.

J.A. Knappett and R.F. Craig. Craig's Soil Mechanics. CRC Press, 8 edition, 2012.

Keng-Wit Lim and José E Andrade. Granular element method for threedimensional discrete element calculations. International Journal for $\mathrm{Nu}$ merical and Analytical Methods in Geomechanics, 38(2):167-188, 2014.

Tang-Tat Ng. Triaxial test simulations with discrete element method and hydrostatic boundaries. Journal of Engineering Mechanics, 130(10):1188 - 1194, 2004. 
Duc-Hanh Nguyen, Emilien Azéma, Philippe Sornay, and Farhang Radjai. Effects of shape and size polydispersity on strength properties of granular materials. Physical Review E, 91(3):032203, 2015.

C Nouguier-Lehon, B Cambou, and E Vincens. Influence of particle shape and angularity on the behaviour of granular materials: a numerical analysis. International Journal for Numerical and Analytical Methods in Geomechanics, 27(14):1207-1226, 2003.

Catherine O'Sullivan. Book review: Craig's soil mechanics, 8th edn, 2013.

John F. Peters, Mark A. Hopkins, Raju Kala, and Ronald E. Wahl. A polyellipsoid particle for non-spherical discrete element method. Engineering Computations, 26(6):645-657, 2009.

Yu Feng Su, Sumana Bhattacharya, Seung Jae Lee, Chang Hoon Lee, and Moochul Shin. A new interpretation of three-dimensional particle geometry: Mavl. Transportation Geotechnics, 23:100328, 2020.

Christian Wellmann, Claudia Lillie, and Peter Wriggers. A contact detection algorithm for superellipsoids based on the common-normal concept. Engineering Computations, 25(5):432-442, 2008.

John R Williams and Alex P Pentland. Superquadrics and modal dynamics for discrete elements in interactive design. Engineering Computations, 9 (2):115-127, 1992.

Beichuan Yan and Richard Regueiro. Comparison between o (n2) and o (n) neighbor search algorithm and its influence on superlinear speedup in parallel discrete element method (dem) for complex-shaped particles. Engineering Computations, 35(6):2327-2348, 2018a.

Beichuan Yan and Richard Regueiro. Large-scale dynamic and static simulations of complex-shaped granular materials using parallel threedimensional discrete element method (dem) on dod supercomputers. Engineering Computations, 35(2):1049-1084, 2018b.

Beichuan Yan and Richard A Regueiro. A comprehensive study of mpi parallelism in three-dimensional discrete element method (dem) simulation of complex-shaped granular particles. Computational Particle Mechanics, 5 (4):553-577, 2018c. 
Beichuan Yan and Richard A Regueiro. Superlinear speedup phenomenon in parallel 3d discrete element method (dem) simulations of complex-shaped particles. Parallel Computing, 75:61-87, $2018 \mathrm{~d}$.

Beichuan Yan and Richard A Regueiro. Comparison between pure mpi and hybrid mpi-openmp parallelism for discrete element method (dem) of ellipsoidal and poly-ellipsoidal particles. Computational Particle Mechanics, 6(2):271-295, 2019a.

Beichuan Yan and Richard A Regueiro. Definition and symmetry of averaged stress tensor in granular media and its $3 \mathrm{~d}$ dem inspection under static and dynamic conditions. International Journal of Solids and Structures, 161: 243-266, 2019b.

Beichuan Yan and Richard A Regueiro. Three-dimensional discrete element method parallel computation of cauchy stress distribution over granular materials. International Journal for Numerical and Analytical Methods in Geomechanics, 43(5):974-1004, 2019c.

Beichuan Yan, Richard A Regueiro, and Stein Sture. Three-dimensional ellipsoidal discrete element modeling of granular materials and its coupling with finite element facets. Engineering Computations, 27(4):519-550, 2010. 